\title{
In ricordo di Vittorio Bianchi
}

Vittorio Bianchi fu Ernesto e fu Mary Sbertoli naque a Cogoleto (Genova) il 17 febbraio 1919. Laureato in Medicina e Chirurgia presso l'Università di Genova nel 1943 con lode fu Allievo interno della Clinica Medica di Genova negli anni 1942 e 1943, Assistente Volontario alla Clinica Medica di Perugia, quindi della Clinica Medica di Genova negli anni seguenti dove fu nominato Assistente di ruo-

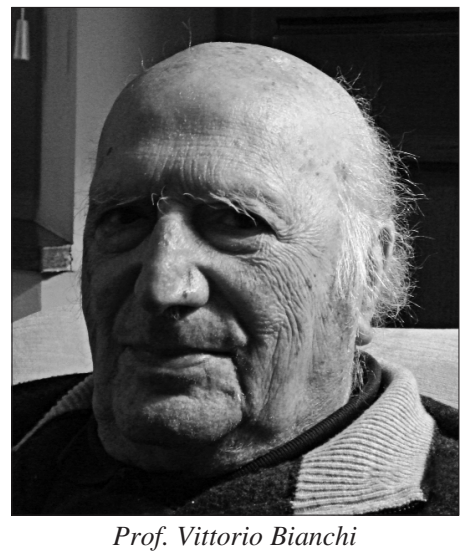

L'attività didattica oltre che nei Corsi di laurea ed in altre occasioni venne svolta da Vittorio Bianchi nella Scuola di Specializzazione in Reumatologia, quest'ultima tra le prime inaugurate in Italia nel 1976.

Promotore delle Leghe Regionali contro le malattie reumatiche, Vittorio Bianchi svolse intensa attività in questo campo con riunioni e conferenze.

lo per concorso nel 1950. Successivamente Assistente di ruolo alla Patologia Medica diretta dal prof. Aminta Fieschi nel periodo 19541969.

Nel 1955 conseguì la libera docenza in Patologia Medica, nel 1958 la libera docenza in Clinica Medica e nel 1962 la libera docenza in Idrologia Medica. Nel 1962 la Facoltà medica di Genova gli conferìl'incarico di Idrologia Medica. Nel 1970 vinse il Concorso per la Cattedra di Reumatologia bandito dall'Università di Roma e nel 1972 gli fu conferita la Direzione della Cattedra di Reumatologia dell'Università di Genova.

L'attività assistenziale inizialmente svolta quale Direttore del Centro Reumatologico dell'Università permise di attivare tra l'altro, la Sezione di Prevenzione del Centro Reumatologico all'lstituto Emilio Bruzzone, come pure servizi di Reumatologia all'lstituto Liberti Poli ed all'lstituto San Giorgio presso le UU.SS.LL Liguri.

Il Centro Reumatologico Istituto Emilio Bruzzone, dotato di sede propria comprendeva Ambulatori, Day Hospital, radiologia, MOC, laboratori di ricerca e di routine, e palestra di riabilitazione, rappresentando a quell'epoca uno dei primi esempi italiani di assistenza reumatologica integrata.
L'attività di ricerca si indirizzò principalmente agli studi sulla fisiopatologia dei tessuti connettivi. Contributi originali riguardarono la responsabilità di fattori piastrinici nella genesi della fibrosi, l'attivazione della reazione collageno-collagenasi, l'azione delle sostanze tioliche sulla fibrosi, le sindromi da lassità connettivale e tanti altri argomenti (fisiochinesiterapia delle malattie reumatiche, terapia termale, ecc.). L'attività di prevenzione costituì un impegno importante con il monitoraggio della osteoporosi postmenopausale e senile mediante la densitometria ossea, eseguita tra i primi in Italia su un grande numero di soggetti.

Vittorio Bianchi fu Presidente della Società Italiana di Reumatologia nel triennio 1983-'86, realizzando in quell'epoca la gestione autonoma di «Reumatismo», giornale ufficiale della Reumatologia Italiana.

Per chi lo ha conosciuto soprattutto negli anni della sua maggiore attività produttiva, quali i suoi allievi e colleghi, rimane il ricordo di una personalità fiera, dotata di speciale comunicatività ed umanità e sempre incline a conoscere ed apprendere quanto di "nuovo e moderno" si affacciava in quegli anni di iniziale sviluppo della Reumatologia.

Maurizio Cutolo Cattedra di Reumatologia, Università di Genova 